\title{
End-to-end Simulation of mmWave Out-of-band Backhaul Networks in ns-3
}

\author{
Qiang Hu, Yuchen Liu, Yan Yan, and Douglas M. Blough \\ Georgia Institute of Technology \\ Atlanta, USA \\ \{qianghu,yuchen.liu,yyan\}@gatech.edu,doug.blough@ece.gatech.edu
}

\begin{abstract}
In this paper, we focus on the end-to-end simulation of millimeterwave (mmWave) out-of-band backhaul networks in ns-3. We first introduce a design for an out-of-band backhaul module in ns-3. A custom backhaul network device is proposed that can be added to nodes to enable out-of-band backhaul transmissions. Since the new backhaul module is still under development, we extended the existing mmWave integrated-access-and-backhaul (IAB) module to enable mmWave out-of-band backhaul simulation and produce some preliminary results on throughput performance. We also modified the existing scheduler in the IAB module to support customizable scheduling for logical links in out-of-band backhaul networks. We used the customizable scheduler to implement an optimal scheduling algorithm from prior work and demonstrate that it increases throughput performance by up to $40 \%$ in certain scenarios.
\end{abstract}

\section{CCS CONCEPTS}

- Networks $\rightarrow$ Network simulations; • Computing methodologies $\rightarrow$ Modeling methodologies.

\section{KEYWORDS}

ns-3, simulation, millimeter-wave, backhaul

\section{ACM Reference Format:}

Qiang Hu, Yuchen Liu, Yan Yan, and Douglas M. Blough. 2019. End-to-end Simulation of mmWave Out-of-band Backhaul Networks in ns-3. In 2019 Workshop on Next-Generation Wireless with ns-3 (WNGW 2019), fune 21, 2019, Florence, Italy. ACM, New York, NY, USA, 4 pages. https://doi.org/10. $1145 / 3337941.3337943$

\section{INTRODUCTION}

In early 2019, the 3GPP release 15 on 5G NR was released [2] and the telecommunication industry began preparing for commercial launches of 5G. Meanwhile, as we are still in the early stages of the $5 \mathrm{G}$ rollout, both industry and academia continue to evolve and innovate new $5 \mathrm{G}$ technologies. Since it is challenging for small research groups to afford expensive $5 \mathrm{G}$ hardware, analytic methods and simulation tools are crucial to $5 \mathrm{G}$ research and development activities. Considering that the simplifications and assumptions made in analytic models limit the generality of the results, simulation

Permission to make digital or hard copies of part or all of this work for personal or classroom use is granted without fee provided that copies are not made or distributed for profit or commercial advantage and that copies bear this notice and the full citation on the first page. Copyrights for third-party components of this work must be honored

For all other uses, contact the owner/author(s).

WNGW 2019, June 21, 2019, Florence, Italy

(C) 2019 Copyright held by the owner/author(s).

ACM ISBN 978-1-4503-7278-7/19/06.

https://doi.org/10.1145/3337941.3337943 tools that can provide accurate results are in great demand. As a result, $5 \mathrm{G}$ millimeter-wave (mmWave) related modules in $\mathrm{ns}-3$ are gaining attention from the research community, because they are expected to be able to evaluate the performance of $5 \mathrm{G}$ mmWave cellular systems in a flexible, accurate and cost-effective way.

Although multiple mmWave modules for ns-3 have been released, most focus on implementing access tier features, while very few touch the backhaul tier. With the pervasive deployment of small cells in dense urban areas for 5G, the backhaul challenge has become critical due to the enormous amount of data that needs to be forwarded between small-cell base stations (BSs), where wired fiber connections may not be available easily due to the cost and construction limits. Moreover, since it has not yet been standardized, there is an opportunity for research on $5 \mathrm{G}$ mmWave backhaul. Note that mmWave backhaul can be categorized into two types, in-band and out-of-band, depending on whether the access and backhaul tiers use the same frequency band or not. Currently, an integrated-access-and-backhaul (IAB, which is in-band) module is available in ns-3; while no solution to the mmWave out-of-band backhaul has been released to date.

In this paper, we introduce our approach to realize end-to-end simulation of mmWave out-of-band backhaul networks in ns-3. In Section 3.1, we propose a novel design for integrating mmWave out-of-band backhaul in ns-3, which includes a custom network backhaul device. In Section 3.2, we describe an extension we have implemented to the existing IAB (in-band backhaul) module to simulate the basic functionalities of out-of-band backhaul. The modified IAB module was used to produce preliminary simulation results, which are reported in Section 3.2.3.

\section{RELATED WORK}

Several modules have been developed for simulating mmWave networks. The most well-known work [6] is from NYU Wireless and University of Padova, which is the first module developed for the end-to-end simulation of mmWave access networks. Within the EPC module from the ns-3 LTE-LENA project[7, 8], the authors added several customized models covering mmWave MAC and PHY layers, and mmWave channels. Since this work focuses on the access tier of mmWave networks, it lacks the ability to simulate backhaul communication between eNBs. To address this issue, researchers from University of Padova have developed a module which can be used to evaluate the performance of mmWave integrated-access-and-backhaul (IAB) networks [9]. An IAB node can directly communicate with an eNB or other IAB nodes. The authors also proposed a distributed scheduling mechanism to support backhaul and access data transmissions in the multi-hop scenario. Although it is the first ns-3 module to support mmWave backhaul, 


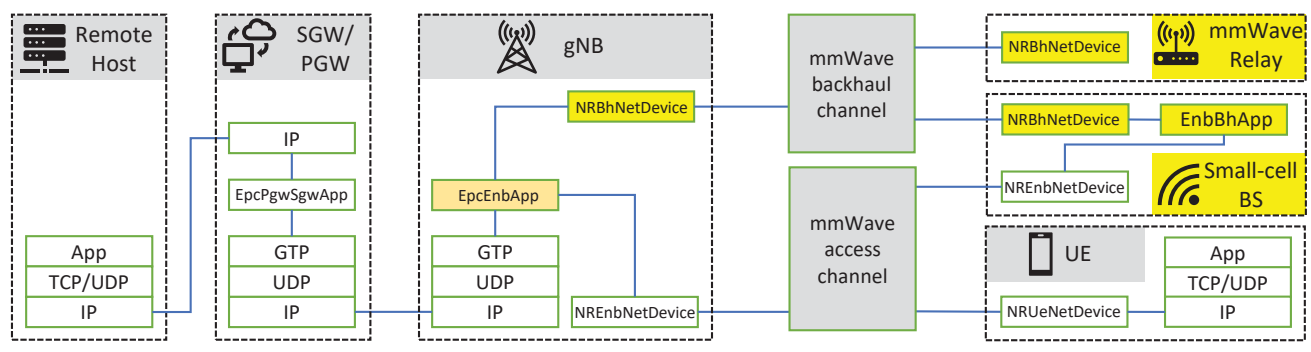

Figure 1: End-to-end Class Overview

it only covers in-band backhaul, where access and backhaul share the same frequency band, and hence it cannot simulate out-of-band backhaul scenarios. Recently, based on the 5G NR standard, CTTC has started the 5G-LENA project [3], the goal of which is to deliver a simulator incorporating fundamental PHY-MAC NR features aligned with NR Release 15 TS 38.300. Similar to [6], currently the 5G-LENA project focuses on the access tier rather than the backhaul tier. As stated above, we are the first to tackle the end-to-end simulation of mmWave out-of-band backhaul networks.

\section{MMWAVE OUT-OF-BAND BACKHAUL SIMULATION}

\subsection{New mmWave Out-of-band Backhaul Module for ns-3}

The ultimate goal of this work is to develop a new module in ns-3 that can be used by the community to simulate mmWave out-ofband backhaul networks and boost research in this area. To align this contribution with industry standards, we plan to build our backhaul module based on the ns-3 5G NR module, being developed by CTTC, which will incorporate fundamental PHY-MAC NR features aligned with NR Release 15 TS 38.300, while other existing mmWave modules lack this feature. In this paper, we provide the high-level architecture of our proposed module design.

Figure 1 provides an overview of the end-to-end classes in our design. The existing and unmodified classes are in white, the only modified class is in gold, and the newly created classes are in yellow. The new NRBhNetDevice, which enables the full functionalities of the mmWave out-of-band backhaul, will be installed into three types of nodes in the simulator, including gNB, small-cell BS, and mmWave relay. Note that the latter two types of nodes will also be introduced for the first time in the ns-3 system. "Small-cell BS" is different from the gNB serving as the macro-cell BS in the $5 \mathrm{G}$ cellular system, because it will not have the wired connection to the SGW/PGW node as gNB does. In fact, mmWave backhaul is proposed because of the existence of a large number of small-cell BSs within each macro-cell in $5 \mathrm{G}$ era and it aims to resolve the issue of forwarding the large amount of backhaul traffic between these small-cell BSs without wired connections. In our design, both NREnbNetDevice and NRBhNetDevice are installed on a small-cell BS node to take care of access and backhaul transmissions, respectively, and the packet exchange between them is realized by the new EnbBhApp class. Since the gNB node should also support the backhaul functionality, an NRBhNetDevice will be installed in it as well. We also plan to modify the EpcEnbApp class so that it can handle packet switching between EPC, access, and backhaul.

As for "mmWave relay" nodes, they are an essential component in relay-assisted mmWave backhaul networks that are deployed to construct a multi-hop line-of-sight (LoS) relaying path between a pair of BSs where the single-hop LoS path is not available due to blockage $[4,5]$. Since relays are simple devices dedicated to the backhaul network, only NRBhNetDevice will be installed on them.

Note that in Figure 1, the mmWave channel class is split into two instances, mmWave backhaul channel and mmWave access channel, to model the "out-of-band" feature of our system. Signals in different channels do not affect each other as they have different frequencies. This differentiates out-of-band backhaul from in-band backhaul, where backhaul links and access links compete for the same channel resource. The existing ns-3 mmWave backhaul module, known as integrated-access-and-backhaul (IAB) module [9], implements the in-band backhaul scenario with a single shared channel.

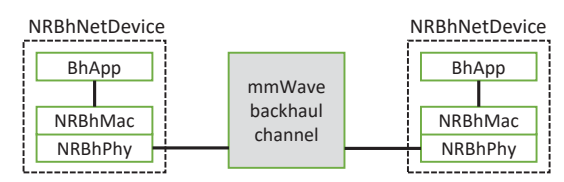

Figure 2: NRBhNetDevice Class Overview

The stack of the proposed NRBhNetDevice is shown in Figure 2. Similar to existing net-devices in the NR module, there are corresponding NRBhMac and NRBhPhy classes, which implement most of the functionalities and features of MAC and PHY layers of NRBhNetDevice. In fact, NRBhPhy can reuse the design of NREnbPhy and NRUePhy. Different from the net-devices in LENA modules, since the backhaul net-device does not need to support any access tier specific functionalities, we choose not to include LteRrc, LtePdcp, or LteRlc classes in our NRBhNetDevice. Instead, we will provide a BhApp class, which implements function blocks that abstract backhaul higher level features such as QoS, guaranteed data transmission, relay path construction and re-configuration, etc.

\subsection{Simulating mmWave Out-of-band Backhaul Using the Existing IAB Module}

While our long-term focus is on development of the dedicated module introduced above, we have also implemented an expedient method to extend the existing mmWave IAB module in ns-3 to 
simulate the basic functionalities of mmWave out-of-band backhaul to get some preliminary results on its throughput performance.

This method is inspired by the fact that the IAB module has already implemented a complete framework for mmWave backhaul simulation, including extending the mmWave eNB in [6] to support backhaul transmissions. Integrating both mmWave UE and eNB netdevices on an IAB net-device to enable both backhaul and access capability was crucial to the fast development of the IAB backhaul module, as it allowed the reuse of mmWave PHY and channel models from [6]. For the same purpose, as we take advantage of the backhaul framework constructed in the IAB module, we could focus on the topology construction and MAC scheduling modifications necessary for out-of-band backhaul without being concerned with other aspects that have been resolved in the IAB module, such as the data bearer set-up along multi-hop backhaul paths.

In the following two subsections, we introduce the details about using mmWave IAB module to set-up the topology for out-of-band backhaul simulations and the modifications to the scheduler to realize the concept of out-of-band backhaul transmissions.

3.2.1 Topology construction. Several network elements need to be deployed in an end-to-end mmWave out-of-band backhaul system, and they can be categorized into two main sets, i.e., the EPC elements and the cellular elements. We directly adopt the EPC module in the ns-3 LTE module as was the case in both [6] and [9]. A remote host connects with the PGW node through a point-topoint wired connection. The $\mathrm{PGW}$ is connected with a mmWave gNB (i.e.,mmWave eNB in [6,9]) through functions defined in EpcEnbApp. The cellular part of the topology starts from the gNB node, which serves as the gateway to the EPC part.

In the cellular part, if the self-backhaul is simulated, where mmWave BSs are connected with each other directly by mmWave wireless links, IAB nodes are installed to serve as the small-cell BSs. In this case, the key difference between the topology of an out-ofband backhaul network and that of an in-band backhaul network in [9] is that no mmWave UE node is deployed to connect with these IAB nodes, except that one mmWave UE node is attached to the IAB node at the end of each path. In fact, that UE also simulates a small-cell BS, which serves as the sink of the backhaul traffic from the gNB. Currently, we are still working on enabling data absorption on the intermediate IAB nodes without attaching access tier UEs, in order to virtually simulate the access tier.

As proposed in $[4,5]$, it is of interest to us to simulate the multihop relaying path of a backhaul logical link, which is constructed by dedicated mmWave backhaul relays between a pair of BSs. In fact, IAB nodes can be deployed as mmWave backhaul relays if no UEs are attached to them, because relays are assumed to be dedicated to the backhaul use, which means no data is consumed at each relay. Starting from the gNB, IAB nodes are connected sequentially with each other, and a UE attached to the last IAB node along the path serves as the destination small-cell BS of a multi-hop backhaul path.

Note that in [9], the attachment of an IAB or UE to a gNB or $\mathrm{IAB}$ is based on a distance metric. The MmWaveHelper class only implemented several "AttachToClosest*" functions, which means a node could only attach to its nearest neighbor. Thus, it does not allow us to freely construct a topology in which the connection construction does not depend on distances. We added helper methods "AttachTo*" to address this issue and allow the attachment to happen according to the input argument of net-device ID.

3.2.2 Scheduler modification. To make the simulation correctly reflect the feature of out-of-band backhaul, we must modify the scheduling within both gNB and IAB net-devices. In the original IAB net-device, the TDMA-based scheduling allocates part of the time resource to access and the rest is assigned to backhaul. In the out-of-band backhaul, however, as we do not need to explicitly simulate the access tier, all the frequency and time resource can be dedicated to the backhaul usage. For an IAB or gNB node without UE attached, all the time slots within a subframe can be allocated for backhaul; while for the last IAB node of each backhaul path, all the time slots can be allocated for transmissions to its attached UE.

In the default scheduler of IAB, "MmWaveFlexTtiMacScheduler", the round-robin style scheduler constrains that backhaul transmissions cannot use more than half of the total available time slots in a subframe, as the other half is reserved for access tier. Our first attempt is to remove this constraint, and to allow all available time slots to be assigned for the backhaul tier. However, due to the highest priority of scheduling retransmissions and the inherent limits from a round-robin scheduler, the throughput performance is not as good as we expected, which is shown later in section 3.2.3.

Actually, in urban environments, small-cell base stations tend to have fixed locations at a higher elevation, which means the topology of mmWave backhaul networks is quite stable. Different from the access links, the channel condition of backhaul links also tends to be stable. Based on these features, we can calculate the optimal schedule of a mmWave backhaul network in the planning stage (the optimal schedule is detailed in [4]) and the pre-calculated optimal schedule can then be pre-loaded into each node in the backhaul network. To do so, we implement a method to install the scheduling decision on each node. We create a new pair of attributes "symAvailStart" and "symAvailEnd" for "MmWaveFlexTtiMacScheduler" to record the range of time slots available for the transmission from the current node to each of its downlink peer node. The time slots assigned for either retransmitting corrupted data or transmitting new data to a specific UE cannot exceed its pre-set range of available time slots. During the simulation, if channel conditions change significantly on some backhaul links, the optimal schedule could change. We are currently developing a method to collect the MCS used on each link as well as a method for the gNB to disseminate the updated optimal schedule to each node.

3.2.3 Example simulations. In this subsection, we provide the results of several example simulations. All simulations used a Manhattan like urban environment, where there are 5 vertical and 5 horizontal intersecting streets, each with a width of 10 meters. The distance between adjacent streets in the same direction was 60 meters. In each blank area surrounded by 4 streets, a $50 \times 50 \mathrm{~m}^{2}$ building with a height of 30 meters was deployed. We set up a gNB node in the center of the area, and IABs or UEs were deployed at street intersections. To simplify the analysis, we set the height of every node to 20 meters, which resulted in a UMi-StreetCanyon scenario since buildings are higher than wireless nodes and most of the secondary interference due to the concurrent transmissions on different links was blocked by those buildings. The MmWave3gppChannel model 
and the MmWave3gppBuildingsPropagationLossModel were used. For traffic generation, we adopted the UdpClientServer application. For each UE node attached at the end of a backhaul path, a UdpServer application was installed, and a UdpClient application was installed at the remote host correspondingly, which had the responsibility to generate UDP packet stream controlled by the Interval attribute. The UDP packet size was 1400 bytes. The backhaul frequency band was set to $28 \mathrm{GHz}$ with a total bandwidth of 1 $\mathrm{GHz}$. The subframe duration was $1 \mathrm{~ms}$ and each subframe contained 24 OFDM symbols. Note that, although a UDP application was used, because the RLC layer was configured with Acknowledge Mode (AM), link-level retransmissions were enabled. With the topology of Figure 3 and the specified parameters, our simulation results incurred a packet loss ratio of close to $0 .^{1}$

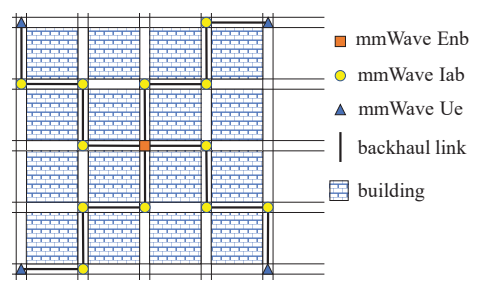

Figure 3: A mmWave Out-of-band Backhaul with 4 Paths

We first simulated the single backhaul path case to see how high a throughput can be achieved with out-of-band backhaul under the default setting of IAB module. If the backhaul path only contained one hop, it is referred to as the case "1-hop"; while if an IAB node was deployed between the gNB and a UE, it is called the "2-hop" case. In both cases, each hop is LoS with a length of 60 meters. Figure 4a shows that as the UDP packet interval was reduced (i.e., "traffic rate" increased), the "1-hop" path reached a maximum end-toend throughput close to 3 Gbps when the packet interval was 3 us, which is slightly below the maximum rate of $3.2 \mathrm{Gbps}$ resulting from the parameters of the IAB module [9] (the MCS had the maximum value of 28 for the transmission in each time slot). However, in the "2-hop" case, the maximum throughput was halved, because an IAB node cannot transmit and receive at the same time.

Figure $4 \mathrm{~b}$ depicts the impact of MAC scheduling on the throughput performance of the entire out-of-band backhaul network. As shown in Figure 3, there are four 4-hop backhaul paths in the topology. The blue bars in Figure $4 \mathrm{~b}$ indicate the end-to-end backhaul throughput of each path, when the round-robin based "MmWaveFlexTtiMacScheduler" in the IAB net-device is modified to support the out-of-band backhaul; while the red bars show the end-to-end backhaul throughput of each path when the optimal scheduling calculated for the topology is pre-installed in the scheduler. The optimal scheduling scheme helps increase the total throughput by about $40 \%$ as compared to the modified round-robin scheduling scheme. Note that in the topology shown in Figure 3, the mmWave Enb connects 4 backhaul links; however, as there is only one radio chain on the Enb, in the optimal scheduling, the 4 links obtain time slots in the numbers of $\{6,5,6,5\}$. That is to say, the PHY rate has been divided into 4 parts almost evenly. If we sum the throughput
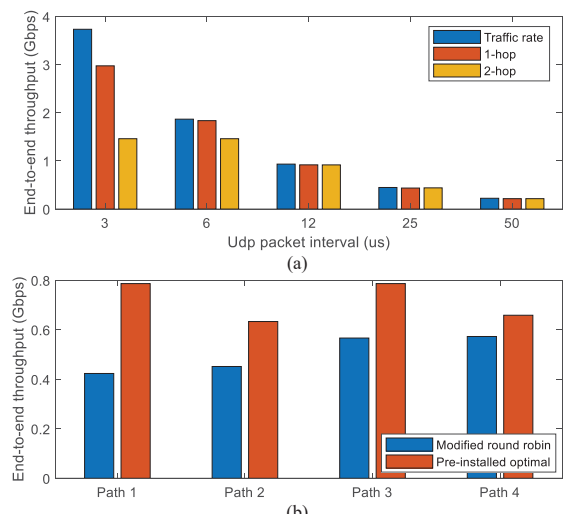

Figure 4: (a) Single-path Scenario (b) Multi-path Scenario

of the 4 paths with optimal scheduling, it gives $2.87 \mathrm{Gbps}$, which is slightly smaller than the maximum throughput of the "1-hop".

\subsection{Changes to the Base mmWave Iab Module}

During our development, we found and fixed several minor problems with the base IaB module:

1) In MmWaveFlexTtiMacScheduler: : CalcMinTbSizeNumSym() the bisection line search does not consider the case that the temporarily calculated tbSize equals to bufSize, and it would result in a wrong Transmission Block (TB) size occasionally.

2) In MmWaveAmc: : GetTbSizeFromMcsSymbols () the calculation of the number of code blocks does not follow the process in [1] exactly. The total size of data should be tbSize + m_crcLen, while each code block contains at most cbSize - m_crcLen bits.

\section{ACKNOWLEDGMENTS}

This research was supported in part by the U.S. National Science Foundation through Award CNS-1813242.

\section{REFERENCES}

[1] 3GPP. 2019. Medium Access Control (MAC) Protocol Specification. Retrieved April 7, 2019 from https://www.3gpp.org/DynaReport/36321.htm

[2] 3GPP. 2019. Release 15. Retrieved April 8, 2019 from https://www.3gpp.org/ release-15

[3] CTTC. 2019. 5G-LENA project. Retrieved April 7, 2019 from https://5g-lena.cttc.es/

[4] Q. Hu and D. Blough. 2017. Relay Selection and Scheduling for Millimeter Wave Backhaul in Urban Environments. In Proceedings of IEEE 14th International Conference on Mobile Ad Hoc and Sensor Systems (MASS '17). IEEE, Orlando, USA.

[5] Q. Hu and D. Blough. 2018. Optimizing Millimeter-Wave Backhaul Networks in Roadside Environments. In Proceedings of IEEE International Conference on Communications (ICC '18). IEEE, Kansas City, USA.

[6] M. Mezzavilla, M. Zhang, M. Polese, R. Ford, S. Dutta, S. Rangan, and M. Zorzi. 2018. End-to-end Simulation of $5 \mathrm{G}$ mmWave Networks. IEEE Communications Surveys \& Tutorials 20, 3 (2018), 27.

[7] N. Patriciello, S. Lagen, L. Giupponi, and B. Bojovic. 2019. An Improved MAC Layer for the 5G NR ns-3 module. In Proceedings of the Workshop on ns-3. ACM, Florence, Italy.

[8] G. Piro, N. Baldo, and M. Miozzo. 2011. An LTE Module for the ns-3 Network Simulator. In Proceedings of the 4th International ICST Conference on Simulation Tools and Techniques (ICST '11). EAI, Brussels, Belgium.

[9] M. Polese, M. Giordani, A. Roy, S. Goyal, D. Castor, and M. Zorzi. 2018. End-toend Simulation of Integrated Access and Backhaul at mmWaves. In Proceedings of IEEE 23rd International Workshop on Computer Aided Modeling and Design of Communication Links and Networks (CAMAD '18). IEEE, Barcelona, Spain.

\footnotetext{
${ }^{1}$ The code is available at: https://github.com/hqfrank/ns3-mmwave-relay
} 